\title{
Asymptotic solutions on multiple solutions arising from laminar flow in a uniformly porous channel with expanding or contracting walls
}

\author{
Qizheng Huang ${ }^{1}$, Lin $\mathrm{Li}^{1 *}$ and Zigen Ouyang ${ }^{1}$
}

"Correspondence:

lilinmath@usc.edu.cn

'School of Mathematics and

Physics, University of South China,

Hengyang, China

\section{Springer}

\begin{abstract}
This paper is concerned with asymptotic solutions of a nonlinear boundary value problem which arises from laminar flow in a uniformly porous channel with expanding or contracting walls. For values of the wall suction Reynolds number, multiple solutions are observed. A method involving the inclusion of exponentially small terms in a perturbation series is mainly considered to obtain two of the solutions analytically. In addition, numerical solutions presented for each case agree well with asymptotic solutions, which illustrates that the asymptotic solutions constructed in this paper are more reliable.
\end{abstract}

Keywords: Laminar flow; Expanding and contracting walls; Singular perturbation method; Exponentially small terms

\section{Introduction}

Studies of porous channel flows have become a topic of focus because of many related applications like in the field of bioengineering and medicine. To name a few, biological fluids are transported through shrinking or expanding containers, synchronous pulsation of porous membranes, simulation of respiratory air cycles, and combustion surface regression model of a solid rocket motor. In 1990, a mathematical model on the viscous flow of Newtonian fluid inside a permeable tube with expanding or contracting cross section was established by Goto and Uchida [1]. In their work, the expansion ratio $\alpha$ and the crossflow Reynolds number Re were introduced to measure the expansion of the pipe and the mass transfer, respectively. Later, Dauenhauer and Majdalani [2] considered the case that laminar flow in a porous channel with expanding or contracting walls and thus established a mathematical model. So far there are some studies on the mathematical model. To list a few, one may count Majdalani et al. [3], Asghar et al. [4], Hang Xu et al. [5] and [6-9]. In fact, for the viscous flow in a porous channel with stationary wall, the earliest researcher can be traced back to Berman [10], who showed that, for constant suction or injection at the walls, the solution of the flow equations can be reduced to a single fourth order nonlinear ODE that includes expansion ratio. In his study, a nonlinear boundary value problem

(c) The Author(s) 2019. This article is distributed under the terms of the Creative Commons Attribution 4.0 International License (http://creativecommons.org/licenses/by/4.0/), which permits unrestricted use, distribution, and reproduction in any medium, provided you give appropriate credit to the original author(s) and the source, provide a link to the Creative Commons license, and indicate if changes were made. 
(BVP) was obtained from the classical Navier-Stokes equations. Meanwhile, for small Re, a regularly perturbed expansion was obtained.

For the above problem, a number of further studies about the existence of multiple solutions of such a BVP follow shortly thereafter. There is the work of Robinson [9], Skalak and Wang [11], Shih [12], Stephen [13]. Recently, when the walls of channel were not motionless, Hang Xu et al. obtained three solutions for large suction using homotopy analysis method (HAM). What is more, the temporal and spatial stabilities were considered in the past because of the existence of multiple solutions of the BVP, where one may count Brady [14], Durlofsky and Brady [15], Sobey and Drazin [16]. Dauenhauer and Majdalani also consider that the search for multiple solutions in the present analysis will be influenced by both Reynolds number $R e$ and expansion ratio $\alpha$. However, in Robinson's paper [9], he considered only the influence of the Reynolds number $R e$, regardless of the expansion ratio $\alpha$. Meanwhile, the inner solution was not modified by Si et al. [17]. Besides, some errors also occur in his paper, for example, in (58) the term

$$
\begin{aligned}
r^{2}= & \frac{\beta}{36}\left(\frac{2 \beta}{\pi \varepsilon^{7}}\right)^{\frac{1}{2}} e^{-\frac{\beta}{2 \varepsilon}}\left[1-\varepsilon(2+3 \alpha)-\varepsilon^{2}\left(\frac{9}{2}+5 \alpha-3 \alpha^{2}\right)\right. \\
& \left.+\varepsilon^{3}\left(-\alpha^{3}-10 \alpha-\frac{301}{24}\right)+\cdots\right]
\end{aligned}
$$

should read

$$
\begin{aligned}
r^{2}= & \frac{\beta}{36}\left(\frac{2 \beta}{\pi \varepsilon^{7}}\right)^{\frac{1}{2}} e^{-\frac{\beta}{2 \varepsilon}}\left[1-\varepsilon(2+3 \alpha)-\varepsilon^{2}\left(\frac{9}{2}+5 \alpha-10 \alpha^{2}\right)\right. \\
& \left.+\varepsilon^{3}\left(-\alpha^{3}-10 \alpha-\frac{301}{24}\right)+\cdots\right] .
\end{aligned}
$$

Based on the above-mentioned work, we have realized the importance of extending previous investigations by presenting theoretical solutions for large suction in a porous channel with expanding or contracting walls. Therefore, a principal objective of the current study is to overcome a deficiency in their model that does not account for modifying the inner solution. Specifically, in Sect. 2, by introducing the flow geometry and governing equations with boundary conditions, a BVP (15)-(16) including two parameters (i.e. Re and $\alpha$ ) is obtained. The numerical solution on multiple solutions are presented in Sect. 3. In Sect. 4 , in order to obtain the asymptotic solution with a large suction Reynolds number, we use a singular perturbation method. An analytic investigation was carried out for larger negative $R e$ in which exponentially small terms were included. In Sect. 5, to get a more accurate solution, we will modify the inner solution and outer solution, which produces dual analytic solutions for large suction. The numerical and analytic results are compared and discussed in Sect. 6. Finally, Sect. 7 concludes the paper.

\section{Mathematical formulation of the problem}

We assume that the channel is of semi-infinite length with one closed end. In addition, to consider a two-dimensional flow, we assume that the distance $2 \alpha$ between the porous walls is much smaller than the channel's width. Both sidewalls are assumed to have equal permeability $-v_{\omega}$ and to expand or contract uniformly by a time-dependent rate $\alpha$. As shown 
Figure 1 Physical configuration

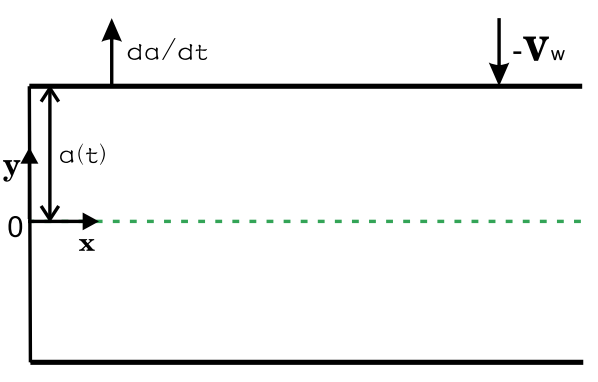

in Fig. 1, $x$ and $y$ indicate the stream wise direction and the normal direction, respectively. $u$ and $v$ denote the velocity components along $x$ - and $y$-axes. The flow velocity is zero at the closed end $(x=0)$. As a result, the motion of a viscous incompressible and electrically conducting fluid through a porous channel with an applied transverse magnetic field can be described by the following equation:

$$
\begin{aligned}
& \nabla \cdot \mathbf{V}=0, \\
& \frac{\partial \mathbf{V}}{\partial t}+(\mathbf{V} \cdot \nabla) \mathbf{V}=-\frac{1}{\rho} \nabla p+\nu \nabla^{2} \mathbf{V}
\end{aligned}
$$

where $\mathbf{V}=(u, v)$ and the symbols $v$ represent the viscosity of the fluid. When we take into account the symmetry with respect to the midsection plan, the necessary boundary for the half-domain may be as follows:

$$
\begin{aligned}
& u=0, \quad v=-v_{w} ; \quad y=a(t), \\
& \frac{\partial u}{\partial y}=0, \quad v=0 ; \quad y=0, \\
& u=0, \quad v=0 ; \quad x=0 .
\end{aligned}
$$

An appropriate stream function $\phi$ is introduced

$$
\phi=\frac{v x}{a} F(\eta, t)
$$

where $\eta=\frac{y}{a}$ is a dimensionless variable. Then the velocity components become

$$
u=v x a^{-2} F_{\eta}, \quad v=-\frac{v}{a} F
$$

Substituting Eq. (9) and Eqs. (5)-(7) and denoting $f=\frac{F}{R e}$, with the assumptions that the wall expansion ratio $\alpha$ is constant and $F$ is made dependent on $y$ and $\alpha$, we can obtain the following equation:

$$
F_{\eta \eta \eta \eta}+\alpha\left(\eta F_{\eta \eta \eta}+3 F_{\eta \eta}\right)+F F_{\eta \eta \eta}-F_{\eta} F_{\eta \eta}-\alpha^{2} v^{-1} F_{\eta \eta t}=0
$$

where $\alpha$ is the wall expansion ratio defined by

$$
\alpha=\frac{a \dot{a}}{v} .
$$


Note that the expansion ratio will be positive for expansion and negative for contraction. An integration of Eq. (10) produces

$$
F_{\eta \eta \eta}+\alpha\left(\eta F_{\eta \eta}+2 F_{\eta}\right)+F F_{\eta \eta}-F_{\eta}^{2}-\alpha^{2} v^{-1} F_{\eta t}=k_{0}
$$

where $k_{0}$ is a space-invariant parameter. The boundary conditions (5)-(7) become

$$
F_{\eta \eta}(0)=0, \quad F(0)=0, \quad F_{\eta}(1)=0, \quad F(1)=R e,
$$

where $R e$ is the permeation Reynolds number defined by $R e=\frac{a v_{\omega}}{v}$. In view of the physical meaning, $R e$ is positive for injection and negative for suction. Let

$$
g=\frac{F}{R e}
$$

a similar solution with respect to both space and time can be developed by the transformation that Uchida and Aoki [18] has described: $\alpha$ is constant and $f=f(\eta)$, which leads to $g_{\eta t}=0$. Under these assumptions, Eq. (12) becomes

$$
g^{\prime \prime \prime}+\alpha\left(\eta g^{\prime \prime}+2 g^{\prime}\right)+\operatorname{Re}\left(g g^{\prime \prime}-g^{\prime 2}\right)=k,
$$

where $k=\frac{k_{0}}{R e}$. The boundary conditions (13) are translated into

$$
g^{\prime \prime}(0)=0, \quad g(0)=0, \quad g^{\prime}(1)=0, \quad g(1)=1 .
$$

\section{Numerical solution of the equations of motion}

Equation (15), subject to boundary conditions (16), is a two-point boundary value problem. Here we use bvp4c of Matlab to solve it. Unless stated otherwise, for all our computations with bvp4c, we use the default relative error tolerance $10^{-3}$ and the default absolute error tolerance $10^{-6}$.

Based on the numerical method proposed in [19], the results of Fig. 2 will be discussed by dividing the figure into three sections, as follows:

1. Section I contains the well behaved solutions for suction;

2. Section II covers the solutions whose centerline velocity is positive but whose axial velocity profiles have a maximum located strictly between the wall and the center of the channel;

Figure 2 Branches of $-f^{\prime \prime}(1)$ vs. Re at $\alpha=-2,0,2$

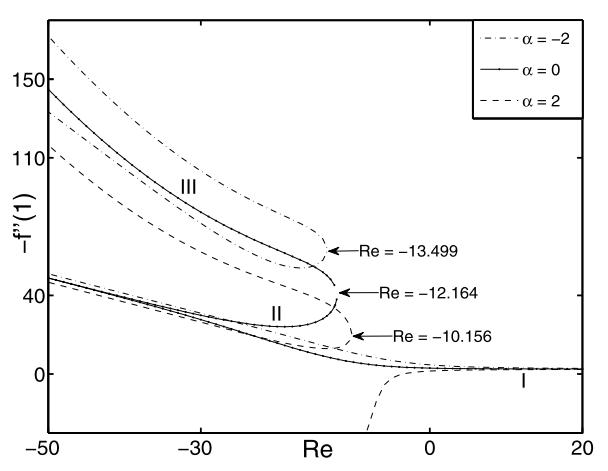


3. Section III includes reverse flow near the center of the channel $(\eta=0)$.

Next, we will mainly concern on asymptotic solutions for Sections I and II solutions. However, Section III solution will not be considered due to the difficulty in solving the corresponding equations with perturbation method. Note that here the interesting mathematics is the way in which the inclusion of exponentially small terms leads to the prediction of dual solutions, which could possibly be extended to predict the Section III solution.

\section{Solution for the large suction Reynolds numbers}

For large suction, Robinson [9] have pointed out the existence of thinning boundary layer when $\alpha=0$. Here we not only consider the boundary layer, but also further extend to the case $\alpha \neq 0$. When $R e \rightarrow-\infty$, the problem (15)-(16) becomes

$$
\varepsilon g^{\prime \prime \prime}+\varepsilon \alpha\left(\eta g^{\prime \prime}+2 g^{\prime}\right)-g g^{\prime \prime}+g^{\prime 2}=\beta^{2}+2 \varepsilon \alpha \beta,
$$

where

$$
\varepsilon=-\frac{1}{R e}, \quad \beta^{2}+2 \varepsilon \alpha \beta=-\frac{k}{R e} .
$$

\subsection{Outer solution}

Considering the conditions

$$
g^{\prime \prime}(0)=0, \quad g(0)=0,
$$

we can know that the outer solution of Eq. (15) is a linear solution, i.e.

$$
g(\eta)=\beta \eta,
$$

where

$$
\beta=\beta_{0}+\beta_{1} \varepsilon+\beta_{2} \varepsilon^{2}+\cdots=\sum_{i=0}^{\infty} \beta_{i} \varepsilon^{i}
$$

Here the coefficients $\beta_{i}(i=0,1,2, \ldots)$ are constants determined later.

\subsection{Inner solution}

To obtain a solution with the viscous layer, an appropriate transformation is introduced:

$$
1-\eta=\varepsilon t .
$$

Combining Eq. (22) with Eq. (17) yields

$$
\varepsilon g^{\prime \prime \prime}-\varepsilon \alpha\left[(1-\varepsilon t) g^{\prime \prime}-2 \varepsilon g^{\prime}\right]+g g^{\prime \prime}-g^{\prime 2}=-\beta^{2} \varepsilon^{2}-2 \alpha \beta \varepsilon^{3},
$$

where the prime (') denotes differentiation with regard to $t$ and the corresponding boundary conditions become

$$
g(0)=1, \quad g^{\prime}(0)=0 .
$$


Because the inner solution meets the condition $g(0)=1$, a perturbation solution will be sought and has the form

$$
g(t)=1+\sum_{r=1}^{\infty} g_{r}(t) \varepsilon^{r} .
$$

From Eq. (24), the relevant boundary condition to be satisfied $g_{r}(t)$ will become

$$
g_{r}(0)=0, \quad g_{r}^{\prime}(0)=0 .
$$

Substituting Eq. (25) into Eq. (23) and equating coefficients $\varepsilon^{r}$, we can obtain

$$
\begin{aligned}
& g_{1}^{\prime \prime \prime}+g_{1}^{\prime \prime}=0, \\
& g_{2}^{\prime \prime \prime}+g_{2}^{\prime \prime}=-\beta_{0}^{2}-g_{1} g_{1}^{\prime \prime}+g_{1}^{\prime 2}+\alpha g_{1}^{\prime \prime 2}, \\
& g_{3}^{\prime \prime \prime}+g_{3}^{\prime \prime}=2 g_{1}^{\prime} g_{2}^{\prime}-g_{1} g_{2}^{\prime \prime}-g_{1}^{\prime \prime} g_{2}+\alpha g_{2}^{\prime \prime}-\alpha t g_{1}^{\prime \prime}-2 \alpha g_{1}^{\prime}-2 \beta_{0} \beta_{1}-2 \alpha \beta_{0}, \\
& g_{n}^{\prime \prime \prime}+g_{n}^{\prime \prime}=\alpha\left(g_{n-1}^{\prime \prime}-t g_{n-2}^{\prime \prime}-2 g_{n-2}^{\prime}-2 \beta_{n-3}\right)+\sum_{i=0}^{\infty}\left(g_{i}^{\prime} g_{n-i}^{\prime}-g_{i} g_{n-i}^{\prime \prime}\right)-\sum_{i=0}^{n-2} \beta_{i} \beta_{n-2-i} .
\end{aligned}
$$

The result of Eq. (27) satisfying the condition Eq. (24) is

$$
g_{1}(t)=A_{1}\left(t-1+e^{-t}\right),
$$

where $A_{1}$ is an arbitrary constant. In a similar way, from Eq. (26), the results for every $g_{r}(t)$ can also be obtained and every $g_{r}(i=1,2, \ldots)$ will include an arbitrary constant $A_{r}$. The first two terms of the inner expansion are

$$
g(t)=1+A_{1} \varepsilon\left(t-1+e^{-t}\right) .
$$

The outer solution Eq. (20) expressed in terms of the inner variable $t$ is

$$
g(t)=\beta_{0}+\left(\beta_{1}-\beta_{0} t\right) \varepsilon+\left(\beta_{2}-\beta_{1} t\right) \varepsilon^{2}+\cdots .
$$

As $t \rightarrow+\infty, e^{-t} \rightarrow 0$, matching the inner solution Eq. (32) with Eq. (33) gives

$$
\beta_{0}=1, \quad A_{1}(t-1)=\beta_{1}-t .
$$

Therefore

$$
\beta_{1}=1, \quad A_{1}=-1 .
$$

Similarly, we can get the solutions of the equations for $g_{1}, g_{2}$ and $g_{3}$ the forms are

$$
g_{2}=4-t+\alpha-e^{-t}\left(\frac{t^{2}}{2}+\alpha t+3 t+4+\alpha\right),
$$




$$
\begin{aligned}
g_{3}= & \frac{129}{4}-(4+\alpha) t+11 \alpha+\alpha^{2}-e^{-t}\left(\frac{t^{4}}{8}+\frac{3 t^{3}}{2}+28 t+9 t^{2}+\frac{65}{2}\right) \\
& -e^{-t}\left(\frac{a t^{3}}{2}+3 \alpha t^{2}+10 \alpha t+11 \alpha+\frac{\alpha^{2} t^{2}}{2}+\alpha^{2} t+\alpha^{2}\right)+\frac{e^{-2 t}}{4}, \\
g_{4}= & \frac{26,377}{72}-\alpha^{3}+21 \alpha^{2}+146 \alpha-\left(\frac{129}{4}+11 \alpha+\alpha^{3}\right) t \\
& -e^{-t}\left(\frac{8963}{24}+\frac{1317 t}{4}+\frac{497 t^{2}}{4}+\frac{82 t^{3}}{3}+4 t^{4}+\frac{3 t^{5}}{8}+8 \alpha^{2} t^{2}+\frac{21 \alpha t^{3}}{2}+\frac{269 \alpha t}{2}\right. \\
& +20 \alpha^{2} t+50 \alpha t^{2}+147 \alpha+21 \alpha^{2}+\alpha^{3} t+\frac{\alpha^{3} t^{2}}{2}+\frac{3 \alpha^{2} t^{3}}{2}+\frac{11 \alpha t^{4}}{8}+\alpha^{3} \\
& \left.+\frac{\alpha^{2} t^{4}}{4}+\frac{\alpha^{3} t^{3}}{6}+\frac{\alpha t^{5}}{8}\right)+e^{-2 t}\left(\frac{57}{8}+\frac{\alpha t}{2}+\frac{t^{2}}{4}+\frac{9 t}{4}+\alpha\right)-\frac{e^{-3 t}}{72} .
\end{aligned}
$$

The coefficients $\beta_{i}(i=2,3, \ldots)$ are given as follows:

$$
\beta_{2}=4+\alpha, \quad \beta_{3}=\frac{129}{4}+11 \alpha+\alpha^{2}, \quad \beta_{4}=\frac{26,377}{72}+146 \alpha+21 \alpha^{2}+\alpha^{3} .
$$

\section{Modified outer solution and inner solution}

For large suction, the solution of the previous section agrees fairly accurately with the numerical solutions. An effort to correctly predict these solution will now be made by investigating the terms neglected in the matching process. Thus we consider modifying the outer expansion these exponentially small terms, because we neglect the terms of the form $t^{r} e^{-r t}$ in the inner expansion. Moreover, the terms have not been matched with any corresponding exponentially small terms in the outer expansion.

\subsection{Modified outer solution}

To get the modified outer solution bearing responsibility for exponentially small terms, satisfying the out conditions, the form of the out solution is assumed to be

$$
g(\eta)=\beta \eta+r f_{1}(\eta)+r^{2} f_{2}(\eta)+\cdots,
$$

where $r$ is exponentially small and $r=o\left(\varepsilon^{r}\right)$, then substituting Eq. (40) into Eq. (17), the forms of $f_{l}(l=1,2, \ldots)$ are

$$
\begin{aligned}
& \varepsilon f_{1}^{\prime \prime \prime}+\varepsilon \alpha\left(\eta f_{1}^{\prime \prime}+2 f_{1}^{\prime}\right)-2 \beta f_{1}^{\prime}-\beta \eta f_{1}^{\prime \prime}=0, \\
& \varepsilon f_{2}^{\prime \prime \prime}+\varepsilon \alpha\left(\eta f_{2}^{\prime \prime}+2 f_{2}^{\prime}\right)-2 \beta f_{2}^{\prime}-\beta \eta f_{2}^{\prime \prime}=f_{1} f_{1}^{\prime \prime}-f_{1}^{\prime 2} .
\end{aligned}
$$

The outer condition Eq. (19), in terms of $f_{r}(\eta)$, is

$$
f_{l}(0)=0, \quad f_{l}^{\prime \prime}(0)=0 .
$$

It is hard to obtain the analytical solution of Eq. (41) and Eq. (42) in a direct way. But if the expansion ratio $\alpha$ is small, a further expansion will be made in $\alpha, f_{1}$ and $f_{2}$ can be expressed as follows:

$$
f_{1}=f_{10}+\alpha f_{11}+o(\alpha),
$$




$$
f_{2}=f_{20}+\alpha f_{21}+o(\alpha)
$$

According to Eq. (43), the corresponding boundary conditions about $f_{i j}(i=1,2 ; j=0,1)$ will be

$$
f_{i j}(0)=0, \quad f_{i j}^{\prime \prime}(0)=0 \text {. }
$$

Substituting Eq. (44) into Eq. (45) and equating coefficients of $\alpha^{0}$ yields

$$
\varepsilon f_{10}^{\prime \prime \prime}+2 \beta f_{10}^{\prime}-\beta \eta f_{10}^{\prime \prime}=0
$$

Differentiation about Eq. (47) gives

$$
\varepsilon f_{10}^{(4)}+\beta f_{10}^{\prime \prime}-\beta \eta f_{10}^{\prime \prime \prime}=0,
$$

where we deduce

$$
f_{10}^{(4)}(0)=0
$$

An extra differentiation of Eq. (48) gives

$$
\varepsilon f_{10}^{(5)}-\beta \eta f_{10}^{(4)}=0
$$

As a result, the solution of Eq. (50) becomes

$$
f_{10}^{(4)}=C e^{\frac{\beta \eta^{2}}{2 \varepsilon}}
$$

Here $C$ is a constant. From (49), $C=0$ is obtained. So the solution of $f_{10}$ is

$$
f_{10}(\eta)=m \eta^{3}+n \eta^{2}+p \eta+q
$$

where $m, n, p$ and $q$ are constants. From (46), we can get $n=q=0$, and substitution of Eq. (52) into Eq. (47) yields $a p=-3 \varepsilon m$. Therefore, the solution for $f_{10}(\eta)$ is

$$
f_{10}(\eta)=\eta^{3}-\frac{3 \varepsilon \eta}{\beta}
$$

Substituting Eq. (45) and Eq. (53) into Eq. (42), we can obtain

$$
\varepsilon f_{20}^{\prime \prime \prime}+2 \beta f_{20}^{\prime}-\beta \eta f_{20}^{\prime \prime}=f_{10} f_{10}^{\prime \prime}-f_{10}^{\prime 2}=-3 \eta^{4}-q^{2} .
$$

Differentiating Eq. (54) twice with respect to $\eta$ gives

$$
\varepsilon f_{20}^{(5)}-\beta \eta f_{20}^{(4)}=-36 \eta^{2}
$$

Satisfying the condition of Eq. (49), the solution is

$$
f_{20}^{(4)}(\eta)=-\frac{36}{\varepsilon} e^{\frac{\beta \eta^{2}}{2 \varepsilon}} \int_{0}^{\eta} s^{2} e^{\frac{-\beta s^{2}}{2 \varepsilon}} d s=-\frac{36}{\varepsilon} e^{\frac{\beta \eta^{2}}{2 \varepsilon}}\left[\int_{0}^{\eta} s^{2} e^{\frac{-\beta s^{2}}{2 \varepsilon}} d s-\int_{0}^{+\infty} s^{2} e^{\frac{-\beta s^{2}}{2 \varepsilon}} d s\right] .
$$


The first integral equation (56) can be calculated, as $R e \rightarrow \infty$ and $\eta \rightarrow 1$, the second can be estimated, the forms gives

$$
\begin{aligned}
f_{20}^{(4)}(\eta) \approx & -\frac{36}{\beta}\left(\frac{\pi \varepsilon}{2 \alpha}\right)^{\frac{1}{2}} e^{\frac{\beta \eta^{2}}{2 \varepsilon}}=-\frac{36}{\varepsilon}(\pi)^{\frac{1}{2}}\left(\frac{\varepsilon}{2 \beta}\right)^{\frac{1}{2}} e^{\frac{\beta}{2 \varepsilon}} e^{\frac{\beta \varepsilon t^{2}}{2}} e^{t-\beta t} e^{-t}, \\
g^{(4)}(\eta)= & \varepsilon^{-4} g^{(4)}(t) \\
= & -\varepsilon^{-3} e^{-t}\left[1-\varepsilon\left(t-\alpha t+2-\frac{t^{2}}{2}+3 \alpha+4 e^{-t} t^{2}\right)-\varepsilon^{2}\left(3 \alpha t^{2}+4 e^{-t}\right.\right. \\
& -\frac{t^{4}}{8}+2 t-7 \alpha^{2}+\frac{9}{2}+3 \alpha^{2} t-4 \alpha t+5 \alpha+\frac{t^{3}}{2}-3 \alpha^{2}-\frac{t^{2} \alpha^{2}}{2}-\frac{\alpha t^{3}}{2} \\
& +\varepsilon^{3}\left(54 e^{-t}+\alpha^{3}+\frac{t^{5}}{8}-\frac{9 e^{-2 t}}{8}-\frac{9 \alpha t}{2}-\frac{t^{6}}{48}-8 \alpha^{2} t^{2}+14 \alpha^{2} t-\frac{\alpha^{3} t^{3}}{6}\right. \\
& -\frac{t^{4}}{4}+\frac{301}{24}+8 \alpha t e^{-t}+7 \alpha t^{2}+\frac{3 \alpha^{3} t^{2}}{2}-\frac{\alpha^{2} t^{4}}{4}+\frac{5 t^{3}}{3}-\frac{7 \alpha t^{3}}{3}+\frac{9 \alpha t^{4}}{8} \\
& \left.\left.+\frac{5 \alpha^{2} t^{3}}{2}+10 \alpha-\frac{7 t^{2}}{4}-3 t \alpha^{3}+20 t e^{-t}+\frac{237 t}{12}+\cdots\right)\right] .
\end{aligned}
$$

Equation (57) includes $e^{-t}$, so $f_{(20)}$ will match with those terms of the form $t^{r} e^{-t}$, in (32), the matching will be taken effect, using the fourth derivative of terms in Eq. (57), which refers to $e^{-t}$, i.e. (58). Matching (57)-(58) yields

$$
\begin{aligned}
r^{2} \frac{36}{\beta}\left(\frac{\pi \varepsilon}{2 \beta}\right)^{\frac{1}{2}} e^{\frac{\beta}{2 \varepsilon}}= & -\varepsilon^{-3}\left[1-\varepsilon\left(t-\alpha t+2-\frac{t^{2}}{2}+3 \alpha+4 e^{-t} t^{2}\right)-\varepsilon^{2}\left(3 \alpha t^{2}+4 e^{-t}-\frac{t^{4}}{8}\right.\right. \\
& +2 t-7 \alpha^{2}+\frac{9}{2}+3 \alpha^{2} t-4 \alpha t+5 \alpha+\frac{t^{3}}{2}-3 \alpha^{2}-\frac{t^{2} \alpha^{2}}{2}-\frac{\alpha t^{3}}{2} \\
& +\varepsilon^{3}\left(54 e^{-t}+\alpha^{3}+\frac{t^{5}}{8}-\frac{9 e^{-2 t}}{8}-\frac{9 \alpha t}{2}-\frac{t^{6}}{48}-8 \alpha^{2} t^{2}+14 \alpha^{2} t\right. \\
& -\frac{\alpha^{3} t^{3}}{6}-\frac{t^{4}}{4}+\frac{301}{24}+8 \alpha t e^{-t}+7 \alpha t^{2}+\frac{3 \alpha^{3} t^{2}}{2}-\frac{\alpha^{2} t^{4}}{4}+\frac{5 t^{3}}{3} \\
& -\frac{7 \alpha t^{3}}{3}+\frac{9 \alpha t^{4}}{8}+\frac{5 \alpha^{2} t^{3}}{2}+10 \alpha-\frac{7 t^{2}}{4}-3 t \alpha^{3} \\
& \left.\left.+20 t e^{-t}+\frac{237 t}{12}+\cdots\right)\right] e^{-\frac{\beta \varepsilon t^{2}}{2}} e^{\beta t-t}
\end{aligned}
$$

However, the terms $t$ and higher powers are not involved in the left-hand side. Therefore, we can cancel terms in $t$ and higher powers in the right-hand side,

$$
\begin{aligned}
r^{2}= & \frac{\beta}{36}\left(\frac{2 \beta}{\pi \varepsilon^{7}}\right)^{\frac{1}{2}} e^{-\frac{\beta}{2 \varepsilon}}\left[1-\varepsilon(2+3 \alpha)-\varepsilon^{2}\left(\frac{9}{2}+5 \alpha-10 \alpha^{2}\right)\right. \\
& \left.+\varepsilon^{3}\left(-\alpha^{3}-10 \alpha-\frac{301}{24}\right)+\cdots\right] \\
= & \frac{\beta^{2}}{36}\left(\frac{2}{\pi \varepsilon^{7}}\right)^{\frac{1}{2}} e^{-\frac{1+\varepsilon^{-1}}{2}}\left[1-\varepsilon\left(\frac{9}{2}+\frac{7 \alpha}{2}\right)-\varepsilon^{2}\left(\frac{57}{4}-\frac{33 \alpha^{2}}{8}+\frac{5 \alpha}{4}\right)\right. \\
& \left.-\varepsilon^{3}\left(\frac{18,313}{144}+\frac{79 \alpha^{3}}{48}-\frac{159 \alpha^{2}}{16}-\frac{23 \alpha}{8}\right)+o\left(\varepsilon^{3}\right)\right] .
\end{aligned}
$$


Clearly, if $\varepsilon$ is so small, the series is positive in the right-hand side. However, Eq. (60) may not offer a good approximation to this critical value of $\varepsilon$, because the coefficients of $\varepsilon^{r}$ in Eq. (60) all have the same sign. The right-hand side of Eq. (60) can be modified by multiplying $\beta^{-6}$, and we can get an alternative series with a better convergence.

$$
\begin{aligned}
r^{2}= & \frac{\beta^{8}}{36}\left(\frac{2}{\pi \varepsilon^{7}}\right)^{\frac{1}{2}} e^{-\frac{1+\varepsilon^{-1}}{2}}\left[1-\varepsilon\left(\frac{21}{2}+\frac{7 \alpha}{2}\right)+\varepsilon^{2}\left(\frac{39}{4}-\frac{33 \alpha^{2}}{8}+\frac{55 \alpha}{4}\right)\right. \\
& \left.-\varepsilon^{3}\left(\frac{15,793}{144}+\frac{79 \alpha^{3}}{48}-\frac{3 \alpha^{2}}{16}-\frac{191 \alpha}{8}\right)+o\left(\varepsilon^{3}\right)\right] .
\end{aligned}
$$

If the terms of $o\left(\varepsilon^{4}\right)$ are now believed to be negligible in Eq. (61), then there are two roots for $r$, provided $\operatorname{Re}<-\left(\frac{21}{2}+\frac{7 \alpha}{2}\right)$,

$$
\begin{aligned}
& r= \pm \frac{1}{6}\left(\frac{2}{\pi \varepsilon^{7}}\right)^{\frac{1}{4}} e^{-\frac{1+\varepsilon^{-1}}{4}}\left[1-\varepsilon\left(\frac{5}{4}+\frac{7 \alpha}{4}\right)-\varepsilon^{2}\left(\frac{253}{32}-\frac{17 \alpha^{2}}{32}+\frac{85 \alpha}{16}\right)\right. \\
&\left.-\varepsilon^{3}\left(\frac{82,621}{1152}-\frac{41 \alpha^{3}}{348}+\frac{261 \alpha^{2}}{128}+\frac{3653 \alpha}{128}\right)+o\left(\varepsilon^{3}\right)\right], \\
&\left.g^{\prime \prime \prime}(\eta)\right|_{\eta=0} \approx\left(\frac{2}{\pi \varepsilon^{7}}\right)^{\frac{1}{4}} e^{-\frac{1+\varepsilon^{-1}}{4}}\left[1-\varepsilon\left(\frac{5}{4}+\frac{7 \alpha}{4}\right)-\varepsilon^{2}\left(\frac{253}{32}-\frac{17 \alpha^{2}}{32}+\frac{85 \alpha}{16}\right)\right. \\
&\left.-\varepsilon^{3}\left(\frac{82,621}{1152}-\frac{41 \alpha^{3}}{348}+\frac{261 \alpha^{2}}{128}+\frac{3653 \alpha}{128}\right)+o\left(\varepsilon^{4}\right)\right] \\
&= \mp\left(\frac{2|\operatorname{Re}|^{7}}{\pi}\right)^{\frac{1}{4}}\left[1+\frac{1}{\operatorname{Re}}\left(\frac{5}{4}+\frac{7 \alpha}{4}\right)-\frac{1}{R e^{2}}\left(\frac{253}{32}-\frac{17 \alpha^{2}}{32}+\frac{85 \alpha}{16}\right)\right. \\
&\left.+\frac{1}{R e^{3}}\left(\frac{82,621}{1152}-\frac{41 \alpha^{3}}{348}+\frac{261 \alpha^{2}}{128}+\frac{3653 \alpha}{128}\right)+o\left(\frac{1}{R e^{3}}\right)\right] .
\end{aligned}
$$

\subsection{Modified inner solution}

The term $r f_{1}(\eta)$ applied for the modified outer expansion is not suit to any terms in the inner expansion. As a consequence, we will modify the inner solution so as to contain the terms that is suit to $r f_{1}(\eta)$, and the form is as follows:

$$
g(t)=1+\sum_{r=1}^{\infty} g_{r}(t) \varepsilon^{r}+r_{1} \sum_{r=0}^{\infty} h_{r}(t) \varepsilon^{r}+o\left(r_{1}\right) .
$$

Besides, the constant $\alpha$ takes two values, i.e., dual solutions, relying on the sign of $r$. So the form should be

$$
\alpha=\sum_{r=0}^{\infty} \alpha_{r} \varepsilon^{r}+r \sum_{r=0}^{\infty} \beta_{r} \varepsilon^{r}+o(r) .
$$

In Eqs. (64)-(65), $\beta_{r}$ and $h_{r}(t)$ must meet Eq. (23) and the boundary conditions Eq. (24), while $r_{1}$ is an exponentially small term with respect to $r$, and the corresponding boundary condition for $h_{r}(t)$ becomes

$$
h_{r}(0)=0, \quad h_{r}^{\prime}(0)=0 .
$$


Considering Eq. (53) and Eq. (65), the first two terms of the modified outer solution Eq. (40) will be

$$
g(\eta)=\sum_{r=0}^{\infty} \alpha_{r} \varepsilon^{r} \eta+r \sum_{r=0}^{\infty} \beta_{r} \varepsilon^{r} \eta+r\left(\eta^{3}-\frac{3 \varepsilon \eta}{\alpha}\right)+\cdots .
$$

In the above, the terms of order $r$ become

$$
r\left[\eta^{3}+\left(\sum_{r=0}^{\infty} \beta_{r} \varepsilon^{r}-\frac{3 \varepsilon}{\alpha}\right) \eta\right] .
$$

To obtain the single term with (68), we set

$$
\sum_{r=0}^{\infty} \beta_{r} \varepsilon^{r}-\frac{3 \varepsilon}{\alpha} \eta=\sum_{r=0}^{\infty} w_{r} \varepsilon^{r}
$$

which makes the modified outer and inner solutions match much simpler and easier. By combining the constant $w$ with the inner variable $t$, (68) will be

$$
\begin{gathered}
r\left[1+w_{0}+\left(w_{1}-w_{0} t-3 t\right) \varepsilon+\left(w_{2}-w_{1} t-3 t^{2}\right) \varepsilon^{2}\right. \\
\left.\quad+\left(w_{3}-w_{2} t-t^{3}\right) \varepsilon^{3}+\left(w_{4}-w_{3} t\right) \varepsilon^{4}+\cdots\right] .
\end{gathered}
$$

We differentiate Eq. (23) about $t$ once again, the form will become

$$
g^{i \nu}+3 \varepsilon^{2} \alpha g^{\prime \prime}+\left(\varepsilon^{2} \alpha t-\varepsilon \alpha\right) g^{\prime \prime \prime}-g^{\prime} g^{\prime \prime}-g g^{\prime \prime \prime}=0 .
$$

Substituting Eq. (64) into Eq. (71) and equating coefficients of $r_{1}$, the equation about $h_{0}(t)$ will be

$$
h_{0}^{i v}(t)+h_{0}^{\prime \prime \prime}(t)=0 .
$$

The corresponding boundary conditions Eq. (66) belong to Eq. (72). So the solution becomes

$$
h_{0}(t)=A_{0}\left(t-1-e^{-t}\right)+B_{0} t^{2},
$$

where $A_{0}$ and $B_{0}$ are constants. Matching with (69) and letting $t \rightarrow+\infty$ in Eq. (73), we can obtain

$$
A_{0} r_{1}(t-1)+B_{0} r_{1} t^{2} \sim r\left[1+w_{0}+\left(w_{1}-w_{0} t-3 t\right) \varepsilon+\cdots\right] .
$$

The terms of $o(r)$ match only if

$$
1+w_{0}=0, \quad \text { i.e. } \quad w_{0}=-1 .
$$

In the same way, the terms of $o(\varepsilon r)$ about (74) show that

$$
r_{1}=r \varepsilon
$$


and

$$
A_{0}=-w_{0}-3=-2, \quad w_{1}=-A_{0}=2, \quad B_{0}=0 .
$$

As a result, the form of $h_{0}(t)$ is as follows:

$$
h_{0}(t)=-2\left(t-1-e^{-t}\right) \text {. }
$$

Next, we will collect the coefficient of $\varepsilon r_{1}$, and the form of $h_{1}(t)$ is

$$
h_{1}^{i v}+h_{1}^{\prime \prime \prime}=\alpha h_{0}^{\prime \prime \prime}+g_{1}^{\prime} h_{0}^{\prime \prime}+g_{1}^{\prime \prime} h_{0}^{\prime}-g_{1} h_{0}^{\prime \prime \prime}-g_{1}^{\prime \prime \prime} h_{0} .
$$

With (79), we can obtain

$$
h_{1}^{i v}+h_{1}^{\prime \prime \prime}=2 \alpha e^{-t}+4 t e^{-t}
$$

The solution of Eq. (80) that is satisfied with the conditions at $t=0$ is

$$
h_{1}(t)=-\left(2 t^{2}+12 t+2 \alpha t+C_{1}+6 \alpha+24\right) e^{-t}+\frac{C_{2} t^{2}}{2}+C_{3} t+C_{4} \text {, }
$$

where $C_{1}, C_{2}, C_{3}$, and $C_{4}$ are constants. Combining Eq. (80) with Eq. (70) and collecting the coefficients of $r \varepsilon^{2}$, we can get

$$
C_{1}=-4 \alpha-10, \quad C_{2}=6, \quad C_{3}=-2, \quad C_{4}=2 \alpha+14,
$$

and therefore

$$
h_{1}(t)=-\left(2 t^{2}+12 t+2 \alpha t+2 \alpha+14\right) e^{-t}+3 t^{2}-2 t+2 \alpha+14 \text {. }
$$

In the same way, the equation for $h_{2}(t)$ is obtained as follows:

$$
\begin{aligned}
h_{2}^{i v}+h_{2}^{\prime \prime \prime}= & -3 \alpha h_{0}^{\prime \prime}-\alpha t h_{0}^{\prime \prime \prime}+\alpha h_{1}^{\prime \prime \prime}+g_{1}^{\prime} h_{1}^{\prime \prime}+g_{2}^{\prime} h_{0}^{\prime \prime}+g_{2}^{\prime \prime} h_{0}^{\prime} \\
& +g_{1}^{\prime \prime} h_{1}^{\prime}-g_{1} h_{1}^{\prime \prime \prime}-g_{2} h_{0}^{\prime \prime \prime}-g_{2}^{\prime \prime \prime} h_{0}-g_{1}^{\prime \prime \prime} h_{1} .
\end{aligned}
$$

The equation for $h_{2}(t)$ is

$$
h_{2}^{i v}+h_{2}^{\prime \prime \prime}=\left(6 \alpha t^{2}+2 \alpha^{2} t-6 \alpha t-4 \alpha-4 \alpha^{2}+3 t^{3}-3 t^{2}-10 t-6\right) e^{-t}+12 e^{-2 t}-6 \text {. }
$$

In a similar way, we can obtain the solution for $h_{2}(t)$, the forms are as follows:

$$
\begin{aligned}
h_{2}(t)= & -4 \alpha^{2}+52 \alpha+\frac{247}{2}-(2 \alpha+14) t-t^{3}-\frac{3}{2} e^{-2 t}-e^{-t}\left[\frac{3}{4} t^{4}+(2 \alpha+8) t^{3}\right. \\
& \left.+(40+15 \alpha) t^{2}+\left(4 \alpha^{2}+50 \alpha+108\right) t-4 \alpha^{2}+52 \alpha+125\right],
\end{aligned}
$$

with

$$
w_{2}=2 \alpha+14, \quad w_{3}=-4 \alpha^{2}+52 \alpha+\frac{247}{3} .
$$




\section{Comparison of the numerical and the modified analytic solutions}

Through comparing two of its main feature with numerical results, we will check on the accuracy of the modified solution. This is not the case in the ordinary numerical, but $g^{\prime \prime \prime}(\eta)$ at $\eta=0$ is

$$
\begin{aligned}
\left.g^{\prime \prime \prime}(\eta)\right|_{\eta=0} \approx & \pm\left(\frac{2}{\pi \varepsilon^{7}}\right)^{\frac{1}{4}} e^{-\frac{1+\varepsilon^{-1}}{4}}\left[1-\varepsilon\left(\frac{5}{4}+\frac{7 \alpha}{4}\right)-\varepsilon^{2}\left(\frac{253}{32}-\frac{17 \alpha^{2}}{32}+\frac{85 \alpha}{16}\right)\right. \\
& \left.-\varepsilon^{3}\left(\frac{82,621}{1152}-\frac{41 \alpha^{3}}{348}+\frac{261 \alpha^{2}}{128}+\frac{3653 \alpha}{128}\right)+o\left(\varepsilon^{3}\right)\right],
\end{aligned}
$$

which, in terms of $R e$, is

$$
\begin{aligned}
= & \mp\left(\frac{2|\operatorname{Re}|^{7}}{\pi}\right)^{\frac{1}{4}} e^{-\frac{1-R e}{4}}\left[1+\frac{1}{\operatorname{Re}}\left(\frac{5}{4}+\frac{7 \alpha}{4}\right)-\frac{1}{\operatorname{Re}^{2}}\left(\frac{253}{32}-\frac{17 \alpha^{2}}{32}+\frac{85 \alpha}{16}\right)\right. \\
& \left.+\frac{1}{\operatorname{Re}^{3}}\left(\frac{82,621}{1152}-\frac{41 \alpha^{3}}{348}+\frac{261 \alpha^{2}}{128}+\frac{3653 \alpha}{128}\right)+o\left(\frac{1}{R e^{3}}\right)\right] .
\end{aligned}
$$

identically zero, for the ordinary outer solution Eq. (20). Combining Eq. (25) with Eq. (39), we can get the numerical results about the inner solution, i.e.

$$
\begin{aligned}
{\left[g^{\prime \prime \prime}(\eta)\right]_{\eta=1} } & =\varepsilon^{-2}\left[g^{\prime \prime}(t)\right]_{t=0} \\
& =-\left[\frac{1}{\varepsilon}-1-\alpha-\left(\frac{13}{2}-\alpha\right) \varepsilon-\left(\alpha^{3}+3 \alpha^{2}+16 \alpha+\frac{677}{12}\right) \varepsilon^{2}+o\left(\varepsilon^{2}\right)\right],
\end{aligned}
$$

which, in terms of $R e$, is

$$
=-\left[-\operatorname{Re}-1-\alpha+\left(\frac{13}{2}-\alpha\right) \frac{1}{\operatorname{Re}}-\left(\alpha^{3}+3 \alpha^{2}+16 \alpha+\frac{677}{12}\right) \frac{1}{R e^{2}}+o\left(\frac{1}{R e^{2}}\right)\right] .
$$

We can get the two results $g^{\prime \prime}(\eta)$ at $\eta=1$ for the modified inner solution Eq. (64), which is a combination of Eq. (91) and a correction because of the exponentially small terms. The correction is

$$
\frac{r \varepsilon}{\varepsilon^{2}}\left[h_{0}^{\prime \prime}(0)+h_{1}^{\prime \prime}(0)+h_{2}^{\prime \prime}(0)\right]+o(r)
$$

which

$$
\begin{aligned}
= & \mp \frac{1}{6}\left(\frac{2}{\pi}\right)^{\frac{1}{4}}|\operatorname{Re}|^{\frac{11}{4}} e^{-\frac{1-R e}{4}}\left[1+\frac{1}{\operatorname{Re}}\left(\frac{5}{4}+\frac{7 \alpha}{4}\right)-\frac{1}{R e^{2}}\left(\frac{253}{32}-\frac{17 \alpha^{2}}{32}+\frac{85 \alpha}{16}\right)\right. \\
& \left.+\frac{1}{\operatorname{Re}^{3}}\left(\frac{82,621}{1152}-\frac{41 \alpha^{3}}{348}+\frac{261 \alpha^{2}}{128}+\frac{3653 \alpha}{128}\right)\right] \\
& \times\left[-2-\frac{2 \alpha+12}{\operatorname{Re}}+\frac{-6 \alpha^{2}+22 \alpha+17}{R^{2}}+\cdots\right]+o(r) .
\end{aligned}
$$

In Table 1 numerical results for $g^{\prime \prime \prime}(\eta)$ at $\eta=0$ are compared with the analytic values obtained by (89). As observed in Table 1, these results appear to be more accurate for Section I (or II) solutions when the value of $|R e|$ is increased. 
Table 1 Numerical values of $g^{\prime \prime \prime}(\eta)$ at $\eta=0$, for various Re in different sections, compared with the analytical formula (89)

\begin{tabular}{|c|c|c|c|c|c|c|c|c|}
\hline \multirow[t]{2}{*}{$\operatorname{Re}$} & \multirow[t]{2}{*}{ Section } & \multicolumn{2}{|c|}{$\alpha=-0.05$} & \multicolumn{3}{|l|}{$\alpha=0$} & \multicolumn{2}{|l|}{$\alpha=0.05$} \\
\hline & & (89) & Numerical & (89) & Numerical & Robinson [9] & (89) & Numerical \\
\hline-28.142 & I & -0.1993 & -0.1064 & -0.1986 & -0.1791 & -0.1984 & -0.1978 & -0.3094 \\
\hline-37.519 & I & -0.0322 & -0.0047 & -0.0321 & -0.0313 & -0.0321 & -0.2320 & -0.2160 \\
\hline-49.008 & I & -0.0029 & 0.0000 & -0.0029 & -0.0029 & -0.0029 & -0.2289 & -0.2109 \\
\hline-55.934 & I & -0.0007 & 0.0000 & -0.0007 & -0.0007 & -0.0007 & -0.2124 & -0.2104 \\
\hline-28.139 & $\|$ & 0.1994 & 0.3571 & 0.1987 & 0.2249 & 0.1986 & 0.1979 & 0.1377 \\
\hline-37.791 & $\|$ & 0.0305 & 0.2058 & 0.0304 & 0.0311 & 0.0303 & 0.0303 & 0.0045 \\
\hline-44.717 & $\|$ & 0.0073 & 0.1992 & 0.0073 & 0.0073 & 0.0073 & 0.0073 & 0.0002 \\
\hline-56.008 & $\|$ & 0.0006 & 0.0000 & 0.0006 & 0.0006 & 0.0006 & 0.0006 & 0.0000 \\
\hline
\end{tabular}

Table 2 Numerical values of $g^{\prime \prime}(\eta)$ at $\eta=1$ for various values of Re compared with the analytic results obtained from (91) with the exponentially small correction (93)

\begin{tabular}{|c|c|c|c|c|c|c|c|c|}
\hline \multirow[t]{2}{*}{$\operatorname{Re}$} & \multirow[t]{2}{*}{ Section } & \multicolumn{2}{|c|}{$\alpha=-0.05$} & \multicolumn{3}{|l|}{$\alpha=0$} & \multicolumn{2}{|l|}{$\alpha=0.05$} \\
\hline & & $\overline{g^{\prime \prime}(1)}$ & Numerical & $g^{\prime \prime}(1)$ & Numerical & Robinson [9] & $\overline{g^{\prime \prime}(1)}$ & Numerical \\
\hline-28.142 & 1 & -25.4335 & -26.0717 & -25.3943 & -25.4998 & -25.396 & -25.3550 & -24.5345 \\
\hline-37.519 & I & -36.0186 & -36.2887 & -35.9709 & -35.9577 & -35.972 & -35.9233 & -34.0197 \\
\hline-49.008 & I & -47.9502 & -47.8963 & -47.9011 & -47.8015 & -47.901 & -47.8519 & -47.7897 \\
\hline-55.934 & I & -54.8382 & -54.8469 & -54.7889 & -54.8047 & -54.789 & -54.7395 & -54.7409 \\
\hline-28.139 & $\|$ & -28.3421 & -29.4494 & -28.2829 & -28.3993 & -28.282 & -28.2237 & -27.7088 \\
\hline-37.791 & $\|$ & -36.9498 & -38.8043 & -36.9171 & -36.2464 & -36.898 & -36.8484 & -36.5494 \\
\hline-44.717 & $\|$ & -43.6865 & -43.5820 & -43.6368 & -43.4384 & -43.636 & -43.5872 & -43.4808 \\
\hline-56.008 & $\|$ & -54.9340 & -54.9216 & -54.8847 & -54.8572 & -54.885 & -54.8353 & -54.8143 \\
\hline-64.296 & $\|$ & -63.2327 & -63.2286 & -63.1833 & -63.1729 & -63.184 & -63.1339 & -63.1232 \\
\hline
\end{tabular}

The numerical results are compared with the analytic results in Table 2, which include the exponentially small correction. As seen in Table 2, the asymptotic solutions agree well with the numerical ones, indicating that our results are valid.

\section{Conclusions}

In this paper, through modifying outer and inner solutions, we have successfully constructed asymptotic solutions for the BVP (15)-(16). Based on numerical solutions with bvp4c, the accuracy of these asymptotic solutions is also easily verified. Here the idea of obtaining multiple solutions with considering exponentially small terms can also better understand the flow phenomenon.

Acknowledgements

The authors are grateful to the University of South China for support.

Funding

None.

Abbreviations

Not applicable.

Availability of data and materials

Applicable.

Competing interests

The authors declare that they have no competing interests.

Authors' contributions

All authors contributed equally to this paper. All authors read and approved the final manuscript. 


\section{Publisher's Note}

Springer Nature remains neutral with regard to jurisdictional claims in published maps and institutional affiliations.

Received: 11 September 2018 Accepted: 31 January 2019 Published online: 18 February 2019

\section{References}

1. Goto, M., Uchida, S.: Unsteady flows in a semi-infinite contracting or expanding pipe with a porous pipe. In: Proceeding of the 40th Japan National Congress Applied Mechanics, vol. 15, pp. 163-172 (1990)

2. Dauenhauer, E.C., Majdalani, J.: Exact self-similarity solution of the Navier-Stokes equations for a porous channel with orthogonally moving walls. Phys. Fluids 15, 1485-1495 (2003)

3. Majdalani, J., Zhou, C.: Moderate-to-large injection and suction driven channel flows with expanding or contracting walls. Z. Angew. Math. Mech. 83, 181-196 (2003)

4. Asdhar, S., Mushtaq, M., Hayat, T.: Flow in a slowly deforming channel with weak permeability: an analytical approach. Nonlinear Anal., Real World Appl. 11, 555-561 (2010)

5. Xu, H., Lin, Z.L., Liao, S.J., Wu, J.Z., Majdalani, J.: Homotopy-based solutions of the Navier-Stokes equations for a porous channel with orthogonally moving walls. Phys. Fluids 22, 053601 (2010)

6. Terrill, R.M., Shrestha, G.M.: Laminar flow through parallel and uniformly porous walls of different permeability. Appl. Sci. Res. 16, 470-482 (1965)

7. Shrestha, G.: Perturbation techniques in laminar flow. Q. J. Mech. Appl. Math. 20, 233-246 (1967)

8. Yunan, S.W.: Further investigation of laminar flow in porous channel. J. Appl. Phys. 27, 267-269 (1956)

9. Robinson, W.A.: The existence of multiple solutions for laminar flow in a uniformly porous channel with suction at both walls. J. Eng. Math. 10, 23-40 (1976)

10. Berman, A.S.: Laminar flow in channels with porous walls. J. Appl. Phys. 24, 1232-1235 (1953)

11. Skalak, F.M., Wang, C.Y.: On the nonuinque solutions of laminar flow through a porous tube or channel. SIAM J. Appl. Math. 34, 535-544 (1978)

12. Shih, K.G.: On the existence of solutions an equation arising in the theory of laminar flow in a uniformly porous channel with injection. SIAM J. Appl. Math. 47, 526-533 (1987)

13. Stephen, M.C.: Analysis of steady flow in a channel with one porous wall or with accelerating walls. SIAM J. Appl. Math. 15, 429-438 (1991)

14. Brady, J.F.: Flow development in a porous channel or tube. Phys. Fluids 27, 1061-1067 (1984)

15. Durlofsky, L., Brady, J.F.: The spatial stability of a class of similarity solutions. Phys. Fluids 27, 1068-1076 (1984)

16. Sobey, J., Drazin, G.: Bifurcations of two-dimensional channel flows. J. Fluid Mech. 171, 263-287 (1986)

17. Si, X.H., Zheng, L.C., Zhang, X.X.: Existence of multiple solutions for the laminar in a porous with suction at both slowly expanding and contracting walls. Int. J. Miner. Metal. Mater. 18, 494-501 (2011)

18. Uchida, S., Aoki, H.: Unsteady flows in a semi-infinite contracting or expanding pipe. J. Fluid Mech. 82, 371-386 (1977)

19. Li, L., Lin, P., Si, X.H., Zheng, L.C.: A numerical study for multiple solutions of a singular boundary value problem arising from laminar flow in a porous pipe with moving wall. J. Comput. Appl. Math. 313, 536-549 (2017)

20. Terrill, R.M.: Laminar flow in a uniformly porous channel. Aeronaut. Q. 15, 299-310 (1964)

\section{Submit your manuscript to a SpringerOpen ${ }^{\circ}$ journal and benefit from:}

- Convenient online submission

- Rigorous peer review

- Open access: articles freely available online

- High visibility within the field

- Retaining the copyright to your article

Submit your next manuscript at $\gg$ springeropen.com 